\title{
DIAGNOSIS OF DISEASE OF THE OTOLITH APPARATUS.*
}

\author{
By Professor R. BÁRÁny, Upsala, translated by Dr Alex. R. \\ TWEEDIE, Nottingham.
}

TILL now it has not been possible to diagnose disease of the otolith apparatus. A short time ago, however, I succeeded in establishing this diagnosis by means of some very simple investigations, and I hope that therewith further developments in the knowledge of the vestibular apparatus will be elaborated.

My remarks are based on the following case which came under my observation at the aural clinic in Upsala:- A woman, aged 27, had suffered with headaches for the past year. The hearing, the caloric reaction on each side, and the neurological condition were normal. Rheumatoid thickening of the neck and shoulder muscles was present. For the last fourteen days attacks of giddiness had occurred. My assistant, Dr Karlefors, was the first to observe that these attacks only took place when the patient was lying on the right side. During the attacks there appeared a marked rotatory nystagmus to the right with an upward-directed vertical component-the nystagmus became purely rotatory on directing the eyes to the right, and purely vertical on directing the eyes to the left. The attack lasted about half a minute and was accompanied with great giddiness and discomfort. If, however, immediately after the cessation of an attack the head were again turned to the right, no such phenomenon could be induced, and it was necessary for the patient to lie on the back or the left side for a little while before these results could be again evoked. So far there is nothing particularly new in the observation. Oppenheim, Bruns, and myself described similar attacks of giddiness and nystagmus many years ago - and I have assumed that these were referable to the semicircular canal system. In this particular case, however, I carried out a series of small experiments which led further.

When I first examined the patient she was lying on the left side with left cheek on the pillow. If now the patient's head were turned towards the right, but not further than the middle line, no giddiness or nystagmus occurred, even although a long pause had intervened after one of her attacks.

* Communicated at the Stockholm Medical Meeting, r2th October 1920, and published in the Acta Oto-Laryngologica, vol. ii., fasc. 4. 


\section{R. Bárány}

On the other hand, nystagmus was immediately induced if the head were inclined even the slightest towards the right. It was thus obvious that the same movement produced no effect in the first instance, but gave rise to a marked nystagmus in the second test. I now made the following control experiment. If the position and not the movement were the causation of the giddiness, it should make no difference how this "Giddiness-Area" was stimulated. I accordingly made the patient sit up and first of all lean the head on the left shoulder. If now after a sufficient pause she quickly raised the head erect no nystagmus was seen. This, however, immediately appeared if she inclined her head towards the right shoulder and thus stimulated the "Giddiness-Area" in the same way as occurred when she turned the head to the right when lying on the back. By this simple test it was, therefore, made clear that it was not movement but position alone of the head which induced the nystagmus, and that the disease must be located in the apparatus which would be thus affected by the consequent pressure.

From investigations carried out by Magnus and de Kleijn in Utrecht lately (Ber. üb. d. ges. Physiologie, Bd. II., I920), it is now clear, that we are here concerned solely with the otolith apparatus. Magnus continued a research which Wittmaack had instituted in I9IO. Wittmaack subjected guinea-pigs to extreme rotation (2000 revolutions per minute) and demonstrated thereafter that the otolithic membrane, whose specific weight is greater than that of the endolymph fluid, was detached, and in the subsequent histological examinations found separated from its nerve endings. Wittmaack had also investigated the function of the vestibular apparatus in these animals, but his research was insufficient for him to draw definite conclusions therefrom.

Magnus repeated the experiments and demonstrated that, in those animals, when the otolith membrane was actually detached, all so-called compensatory eye movements (that is the reflex of position) were absent, whilst the movement reflex (that is the semicircular canal reaction) was present. If the results of this research, which up till now have not been fully published, are regarded as indisputable - of which I have no doubt-it is now established that the compensatory eye movements are referable to the otolith apparatus. In men it is only possible generally to observe one aspect of this compensatory eye movement - namely, the rolling of the 


\section{Disease of the Otolith Apparatus}

eyes in the opposite direction to the inclination of the head. This opposite-rolling reflex I studied in 1906 and elaborated a special apparatus for its accurate measurement. At that time, however, I came to the conclusion that this reflex also could be attributed to the semicircular canals - this only entailed the assumption that the cupulæ which rest on the cristæ of the ampullæ of the semicircular canals had a different specific weight from the endolymph, in order to support the possibility or rather the probability that these reflexes were referable to the semicircular canals. Such explanation is now condemned by the result of the research of Wittmaack and more especially Magnus, and we must now accept the "opposite-rolling" of the eyes as an otolith reflex. Thus, too, the investigation of this phenomenon becomes of added interest.

I would take this opportunity to mention that, in I9I3, I described a phenomenon which has some bearing on the matter in question. This referred to a patient with multiple sclerosis in whom a left-directed horizontal nystagmus could be induced by laying him on his right side, which nystagmus however-in contradistinction to the particular form of nystagmus to-day described-did not cease after half a minute, but continued so long as the patient remained in this position (Monat.f. Ohrenh., 1913, p. 481), vide infra. Similar phenomena are observable in rabbits, a fact which at once prompted me to give attention to these symptoms in men. Rothfeld, once a pupil of mine and now a neurologist in Lemberg, has discovered that rabbits under the influence of acute alcohol intoxication developed a horizontal nystagmus if laid on the side, and at that particular period of the intoxication when the compensatory eye movements were inhibited with placing them in this position (Arb. aus d. neurol. Institut., Wien, Bd. xx., 1912). Having regard to these experiments, I presume also that in patients with multiple sclerosis the ataxia would cease if the head were inclined towards the right [? or left] shoulder. I have not yet had the chance of testing this point. It must now be our duty to observe if these attacks of nystagmus on quick movement of the head are always referable to the otolith apparatus, and more especially to determine which particular symptoms in the pathology of the vestibular symptoms are really attributable to the otolith apparatus.

[The case of multiple sclerosis referred to by Bárány was described by him at the Meeting of the Austrian Otological 


\section{R. Bárány}

Society held on 27th January I9I3, under the title of "Constant Variation of Spontaneous Nystagmus in accordance with Variation in the Position of the Head."]

The case, which I now wish to bring before you, shows a phenomenon, which, so far as I know, has not yet been described in detail in men.

The patient who suffers from multiple sclerosis, has, with the erect position of the head, a coarse, horizontal rotary nystagmus to the left, when the eyes are directed to the left; and a fine nystagmus to the right, when the eyes are directed to the right. There is no by-pointing, the caloric reaction on both sides is prompt; but no obvious rotary component of the nystagmus to the right appears with cold water irrigation of the left ear. Rotation affords an analogous nystagmus. With the head inclined backwards, rotation induces only slight rotary nystagmus to the right, but a very marked rotary nystagmus to the left. After ten rotations to the right, with the head bent backwards, there appears, first (for a few seconds), a rotary nystagmus to the right, which is then followed by an obvious marked rotary after-nystagmus to the left. Rotation to the left affords a marked rotary after-nystagmus to the left, but no rotary after-nystagmus to the right. The hearing is normal.

The new phenomenon, to which I would now direct your attention, exists in the fact that with the eyes directed forwards, the patient shows no (or only exceedingly slight) nystagmus to the left, but, if the head be inclined to the right (and even when this action is carried out quite slowly and gradually) there appears a fairly marked horizontal nystagmus, with a slight rotary component towards the left, which, if the head is maintained in this position, continues undiminished for a long time. I have repeatedly witnessed this unaltered action for five minutes. The same phenomena are induced if the patient, when lying on his back, turns quite slowly on his right side. The nystagmus is accompanied by by-pointing of both arms to the right and slight giddiness. If the head is inclined to the left a much weaker but still definite horizontal nystagmus towards the right is induced, which in the same manner is maintained undiminished and is accompanied by definite, but less marked, by-pointing of both arms to the left.

Such continuation of nystagmus, resulting from change of the position of the head, has not yet been described in men. I have, however, already seen a second similar case, and as far as my memory serves me, would expect that these cases are 


\section{Disease of the Otolith Apparatus}

not so uncommon. Attention has 'not been directed to this condition up till now, and it is my belief that most of these "continuing" variations of nystagmus have been confused with the shorter attacks of nystagmus, the result of quick movements of the head. Nystagmus attacks, following quick head movements, were first observed and described by Bruns and Oppenheimer. Bruns considered, however, that they should be regarded as a symptom of cysticercus infection of the fourth ventricle. Oppenheimer describes them as symptoms of cerebellar tumour. I have found them, however, just as much in association with circumscribed labyrinth disease as with multiple sclerosis, cerebellar tumours, and cysticercus of the fourth ventricle. These attacks and their probable explanation I have myself already described. I regarded them as due to the non-occurrence of some inhibition, and considered that the nystagmus, elicited on moving the head, thus continued even after this movement ceased. These attacks however differ, in that the nystagmus so caused, in a short time disappeared; whilst in the case in question we have to do with a "continuing" nystagmus.

My attention was directed to the possibility of such nystagmus by the animal tests of Rothfeld. Rothfeld observed that a "continuing". horizontal nystagmus occurred in rabbits, under the influence of alcohol, when laid on the side; the nystagmus was directly associated with paralysis of the normal deviation, which resulted from the position of the animal on its side.

A "continuing" nystagmus, which is dependant on the position of the head and varies with the position of the head, can only be due to a definite "continuing" stimulus, dependant on such position of the head; and such stimulus can be no other than the action of gravity; as would be the effect of pressure on the cupulæ and otoliths. But what is the reason that this nystagmus is not seen in normal people? Can it be that intoxication, or in my case, disease, must first invoke the occurrence of such stimulus? No, certainly this stimulus must be continually present in normal people-that it does not cause nystagmus can only be referred to the fact, that in normal people it is balanced. The stimulus which causes, for example, a left-directed nystagmus, is balanced by an opposing action inducing nystagmus to the right. Intoxication, that is disease, must thus either paralyse the opposing nystagmus or enhance the stimulus producing nystagmus to the left. In rabbits Rothfeld was indeed able to observe a paralysis, that is a paralysis of 


\section{R. Bárány}

deviation, which synchronised with the occurrence of the nystagmus, due to the lateral position. In my patients I have not been able to detect such obvious paralysis; it is, however, possible that the rolling of the eyes in the opposite direction on inclination of the head, is paralysed, but this at present is pure conjecture. A further question is, with what particular nervous route is this paralysis associated?

With the nystagmus to the left, in my case, by-pointing with both arms, corresponding to the nystagmus, is present; thus the necessary stimulus must arise in the ganglion vestibulare of Scarpa, that is the peripheral end organs, if Cajal is correct.

If we suppose that the stimulus does exist in normal people, and that in pathological cases the balance is uncontrolled owing to the absence of an antagonistic stimulus, we could conclude that the antagonistic stimulus must be controlled at its origin, that is, in the ganglion vestibulare; in that case, such control would lie wholly within the extension of Deiter's nucleus, that is Bechterew's nucleus. By this hypothesis the occurrence of the nystagmus can be explained, but not however the occurrence of the associated by-pointing. We are therefore constrained either to assume that the paralysis has its origin in the ganglion vestibulare of the vestibular nerve, or that a paralysis of Deiter's nucleus depresses by centrifugal action the excitability of the corresponding cells in the ganglion vestibulare.

In this connection I should like to acknowledge my thanks to Professor Dr H. Schlesinger, who has repeatedly investigated pathological foci of the acoustic nerve in cases of multiple sclerosis.

You will see how deep an insight into this complicated mechanism is demanded for an accurate study of these phenomena. Certain conclusions will not be reached until we know, after exact confirmation of Cajal's account, the anatomical condition of the parts concerned, and we may be able also to adduce explanations by accurate histological research on pathological cases under observation.

[It may also be valuable for the reader, especially interested in this subject, to refer to the proceedings of the Meeting of the Austrian Otological Society 3oth May I9Io, Monats. f. Ohren., vol. 44, No. 6, or a translation of these reports, published in The Journal of Laryngology, Rhinology, and Otology, I91 I, page 157, et seq. - A. R. T.] 